\title{
The Utility of Diffusion Weighted Imaging and Dynamic Contrast Enhanced MRI Techniques in Differentiation Between Benign and Malignant Uterine Masses
}

\author{
Al-Shimaa Magdy Ammar ${ }^{1}$, Mohammed Mahmoud Dawoud ${ }^{1}$, Mohamed Mohamed Hefeda ${ }^{1}$, \\ Manal Ezzat Badawy ${ }^{1}$, Essmat Hamdy Abozaid ${ }^{2}$ \\ ${ }^{1}$ Radiology Department, Tanta University, Tanta, Egypt \\ ${ }^{2}$ Department of Obstetric and Gynecology, Faculty of Medicine, Tanta University, Tanta, Egypt
}

Email address:

alshimaaammar87@yahoo.com (Al-Shimaa M. A.), Dr.m.dawoud82@gmail.com (M. M. Dawoud), mohamedhefeda@yahoo.com (M. M. Hefeda),Dr.manalezzat1@yahoo.com (M. E. Badawy),

Dr.esmatabouzaid@gmail.com (E. H. Abozaid)

\section{To cite this article:}

Al-Shimaa Magdy Ammar, Mohammed Mahmoud Dawoud, Mohamed Mohamed Hefeda, Manal Ezzat Badawy, Essmat Hamdy Abozaid. The Utility of Diffusion Weighted Imaging and Dynamic Contrast Enhanced MRI Techniques in Differentiation Between Benign and Malignant Uterine Masses. International Journal of Medical Imaging. Vol. 7, No. 3, 2019, pp. 66-80. doi: 10.11648/j.ijmi.20190703.12

Received: July 12, 2019; Accepted: August 15, 2019; Published: September 30, 2019

\begin{abstract}
The aim of the present study was to evaluate the role of diffusion weighted imaging (DWI) and dynamic contrast enhanced magnetic resonance imaging (DCE-MRI) in the differentiation between benign and malignant endometrial, uterine and cervical masses. The study included 78 patients. All patients had uterine or cervical masses on ultrasound examination. All patients had conventional MRI, DWI, and DCE-MRI. The patients were classified into three groups: patients with endometrial masses, myometrial lesions and cervical masses. The mean ADC value of benign endometrial and myometrial lesions was higher significantly from malignant lesions ( $\mathrm{P}$ value $<0.001$ and $<0.001$ ). The cut off value of $\leq 1.1 \times 10^{-3} \mathrm{~mm}^{2} / \mathrm{sec}$ as a predictor of malignant lesions yielded a sensitivity, specificity, PPV, NPV and accuracy of $89.65 \%, 91.54 \%, 88.67 \%, 92.41 \%$ and $95.21 \%$ respectively. The semi-quanitative parameters of DCE-MRI including the enhancement amplitude (EA), maximum slope (MS) and time of half rising (THR), all showed significant difference between the benign and malignant lesion in endometrial, myometrial and cervical lesions. In clinically and sonographically indeterminate endometrial, myometrial or cervical masses, DWI and DCE-MRI proved to be more accurate than conventional MRI in differentiation between benign and malignant masses, with the DWI and ADC value had more sensitivity and specificity especially in endometrial masses and should be included in the routine pelvic MRI. DCE-MRI should be reserved for still questionable cases after DWI and to study the vascularity of the lesions.
\end{abstract}

Keywords: Uterine Masses, Endometrial Masses, DWI, Dynamic Contrast MRI, Functional MRI

\section{Introduction}

Globally, Cancer uterine body and cervix ranked as the 5th and 3rd most common malignancies in females respectively $[1,2]$. In Egypt, uterine cancer is ranked as the 10th most common cancer with increasing incidence in recent years $[3$, 4]. Most uterine cancers arise from the endometrium $(90 \%)$ and only $8 \%$ arise from the myometrium [4].

Ultrasound, which used to be the initial screening investigation for imaging of uterine lesions, has considerable limitations including restricted field of view and overlap in sonographic picture between benign and malignant masses [5-7]. Magnetic resonance imaging (MRI) is the second line technique for diagnosis of uterine masses and differentiation between the benign and malignant masses especially in large masses [8]. Diagnosis of endometrial carcinoma or myometrial sarcoma on MRI depends on the presence of moderate to high T2 signal intensity, which can be seen in the benign lesions like degenerating leiomyoma and benign polyps [9]. Thus the differentiation between benign and malignant uterine masses may be difficult on conventional MRI. 
Diffusion weighted imaging (DWI) is a functional MRI technique, depends on Brownian motion of water molecules, with the signal intensity influenced by factors like number of cells, viscosity of intracellular and extracellular spaces, and tortuosity of the extracellular spaces,. The proton motion decreases in neoplastic and inflammatory processes $[9,10]$. Apparent diffusion coefficient (ADC) is the quantitative derivative of the DWI and known to influenced by cellular density and nucluear to cytoplasmic ratio in solid tissues [1114]. Low $\mathrm{ADC}$ values in malignant tissues compared to benign lesions has been described in different organs [15-18]. The accuracy of DWI in uterine and cervical masses have been previously reported $[19,20]$, Also, some authors have attempted $\mathrm{ADC}$ value measurement in the uterine myomas $[21,22]$.

Dynamic contrast enhanced MRI (DCE-MRI) depends on the rapid acquisition of T1WI in different phases to assess vasculaity and permeability of tissues [23]. The enhancement pattern in DCE MRI reflects the intratumoral vascularity, which provides physiological and anatomical details useful for treatment planning [24]. Dynamic contrast enhanced- MRI depends on the availability of fast MRI sequences obtained before, during and after rapid intravenous injection of gadolinium based contrast media. Interpretation of DCE-MRI data can be quantitative or semi-quantitative $[25,26]$.

The aim of this study is to evaluate the diagnostic accuracy of diffusion weighted imaging and dynamic contrast enhanced MRI in differentiation between benign and malignant uterine and cervical masses.

\section{Patients and Methods}

\subsection{Study Population}

This cross sectional prospective study included seventy eight patients with uterine masses. Institutional research ethics committee approved the study and signed informed consent was obtained from all patients. The study was conducted from October 2016 to March 2019. The final diagnosis of all patients and the mean age in each group is summarized in table 1. All diagnosis was histopathologically proves after myomectomy (13 patients), hysterectomy (42 cases), hystrectmy with bilateral salpingeo-oophrectomy (15 cases) or radical hysterectomy ( 8 cases). Fifty one $(65.4 \%)$ were pre-menopausal and 27 (34.6\%) were post-menopausal.

The exclusion criteria of this study included patients with myomas $<2 \mathrm{~cm}$, patients with no histopathological confirmation and patients with contraindication to MRI examination or contrast.

\subsection{MRI Protocol}

In the current study we used wither Toshiba vantage titan 1.5 Tesla \& GE signa explorer 1.5 Tesla (General Electric Healthcare, Milwaukee, USA) MRI units with the same protocol parameters. The sequences and parameters are summarized in table 2. Patients were asked to fast for 4 hours, all patients received intravenous antispasomid drug (10 mg tiemonium methylsulfate; Visceralgine) to inhibit intestinal movement. All sequences was obtained with phased array surface coil.

\subsection{Diffusion Weighted MRI}

Diffusion weighted imaging was acquired in all patientsin the axial or the sagittal plane prior to administration of contrast medium by using a single shot echo-planar imaging sequence with multipleb values. The $b$ values was 0 , $500,800,1000 \mathrm{~s} / \mathrm{mm}^{2}$ ) in axial protocol and only two values in sagittal focused protocol $\left(500 \& 1000 \mathrm{~s} / \mathrm{mm}^{2}\right)$.

\subsection{Dynamic Contrast Enhanced MRI Study}

post contrast T1 fat sat LAVA Flex images (on GE signa explorer 1.5 Tesla) $\mathrm{O}$ post contrast $\mathrm{T} 1$ fat sat THRIVE (High Resolution Isotropic Volume Examination) on TOSHIBA vantage titan 1.5 Tesla were obtained immediately after rapid intravenous injection of $0.1 \mathrm{mmol} / \mathrm{kg}$ of Magnivist (Dadopentate dimeglumine, Schering, Berlin, Germany) which was followed by injection of $20 \mathrm{~mL}$ of normal saline flushing the tube. Images were obtained sequentially at $0,30,60,90$ and 120 seconds, The parameters was as follows: Field of view $40 \mathrm{~mm}$, slice thickness 4mm, 3D thickness 4, slice gap0mm, number of slices 84 , TR $4.4 \mathrm{~ms}$, TE $2.1 \mathrm{~ms}$, flip angle 12 degrees, slice spacing $0 \mathrm{~mm}$. Also, delayed axial T1 WI with the following parameters (TR/TE:450-650 $\mathrm{msec} / 10^{-1} 6 \mathrm{msec}$, slice thickness $7 \mathrm{~mm}$, slice spacing $1.5 \mathrm{~mm}$ ), delayed sagittal post contrast T1WIwith the following parameters (TR/TE: 450-650/10-16ms; slice spacing $1 \mathrm{~mm}$, slice thickness $5 \mathrm{~mm}$, number of slices 25 ) and post contrast delayed coronal T1WI (TR/TE: 450$650 / 10^{-1} 6 \mathrm{~ms}$; slice spacing $1 \mathrm{~mm}$, slice thickness $5 \mathrm{~mm}$, number of slices 25 ).

\subsection{Interpretation of MRI}

The MRI images were reviewed for the following: size and location of the lesion, contour of the lesion (regular or irregular), signal intensity on different sequences, presence of hemorrhage, cystic changes or fat. Thickness of the endometrium, myometrial infiltration, cervical stromal infiltration. Presence of infiltrated pelvic or para aortic lymph nodes. Involvement of other pelvic organs.

The T2 Signal intensity was interpreted in relation to gluteal muscle, a low T2 SI means that the SI of the lesion was similar to the gluteal muscle and an intermediate T2 SI when the signal intensity is higher than gluteal muscle. The signal intensity of the lesion was said to have high T2 SI when it is higher than the outer myometrium.

\subsection{Interpretation of $\mathrm{DWI}$}

Qualitative analysis: The lesion was either with low signal intensity on diffusion images with high signal in the corresponding ADC maps (facilitated) or show high signal 
intensity on diffusion images with low signal in the corresponding ADC maps (restricted). On DWI, the signal intensity was classified as high when it its equal or higher than the signal intensity of the endometrium.

Quantitative analysis: Regarding the quantitative analysis of DWI, we generated the ADC map on commercially available work station, and then we selected the region of interest manually (the ROI was set as large as possible within the confines of the tumor) which was then automatically calculated on the work station to get the ADC values. For each lesion, the ADC values were calculated three times and the average of the measures was calculated and recorded. ROI were placed away from areas of necrosis and cystic changes.

\subsection{Interpretation of DCE-MRI}

Analysis of Dynamic Contrast-enhanced MR Images

Dynamic data analyze in consensus at a workstation. The entire mass is included in all phases of dynamic run acquisition at 120 second after gadolinium injection. A region of interest (ROI) is manually drawn over the most avidly enhancing mass of the lesion that can be helped by color map. Signal intensity (SI)-time curve was generatd.

There were three types of time intensity curve Type I (gradual rising curve), Type II (plateau curve) and type III (rapid rising curve and rapid wash out).

Semi-Quantitative Dynamic Contrast-enhanced MR Imaging.

Multiple values calculated automatically on the work station after ROI Drawn at the most enlaced region of the mass, through which detected EA (enhancement amplitude), Ms (maximum slope of the curve) and THR (time of half rising).

The mean of the values was calculated for benign, border line and malignant masses.

\subsection{Statistical Analysis}

Mean values and standard deviation were calculated for the parameters studied. The sensitivity, specificity, PPV, NPV, and overall diagnostic accuracy for the DWI and DCE-MRI were calculated. Chi square test and Fisher exact test were used to compare two by two tables in small samples. The Mann-Whitney $U$ test was used for the comparison between variables and $p$ value acquisition The receiver operating characteristic curve (ROC) was generated for different parameters and the areas under the ROC were calculated (Figure 1). The extended McNemar $\mathrm{x} 2$ testwas used to compare the statistal significance between the sensiticity and specificity of different techniques. Associations between assessment of uterine masses and possible pitfalls previously mentioned on $\mathrm{T} 2 \mathrm{~W}$ images were examined by implementing Fisher exact test. P value $<0.05$ was considered statistically significant. All analyses were performed using SPSS Version 14 and StatExact 4.

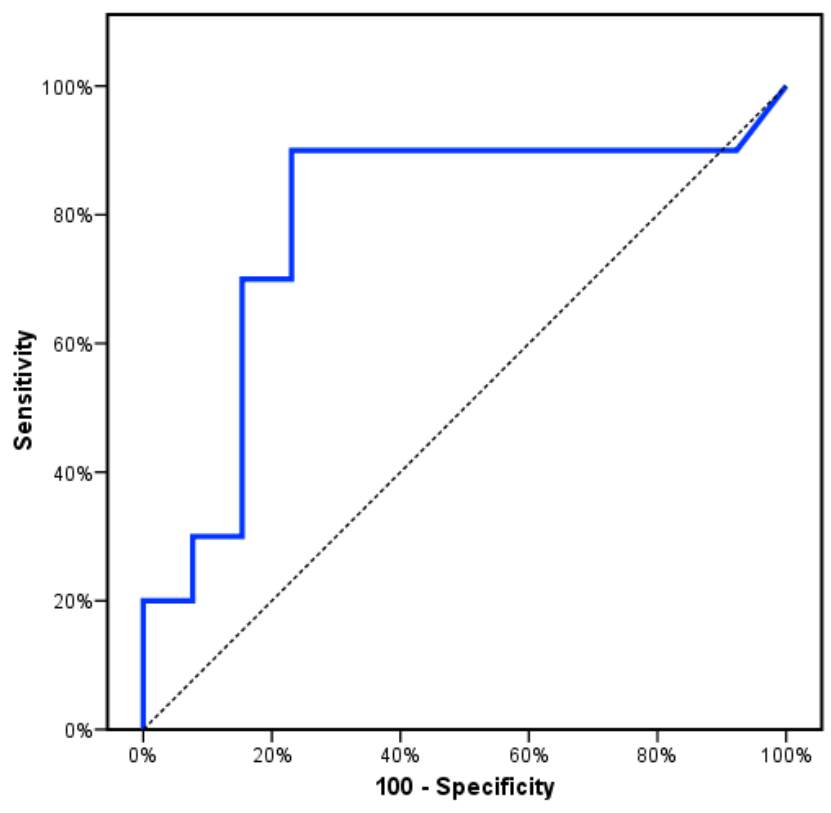

Figure 1. ROC curve for ADC to predict malignant cases in uterine masses.

AUC: Area Under a Curve

$P$ value: Probability value

CI: Confidence Intervals

\section{Results}

The mean age of patients with malignant masses was significantly higher than patients with benign masses $(50.4+4.2$ years VS 39.3+3.5years, $P$ value $<0.001)$.

\subsection{MRI Lesion Characteristics (Table 3)}

The presence of heterogeneous or high T2 signal intensity was more common inmalignant endometrial (Figure 2), myometrial and cervical masses than benign masses $(80 \%$ of malignant endometrial lesions VS $31.4 \%$ of benign lesions, $83.4 \%$ of malignant myometrial lesions Vs $17.4 \%$ of benign lesions and $100 \%$ of malignant cervical lesions Vs $23 \%$ of benign cervical lesions), with the difference statistically significant $(\mathrm{P}<0.05)$. Also, irregular lesion outline was noted in $100 \%$ of malignant cervical masses and in only $15 \%$ of benigncervical masses. Also irregular outline was more common in malignant endometrial and myometrial masses (90\% and $66.7 \%$ respectively) than benign lesions $(9.1 \%$ and $17.4 \%$ respectively). Also, the presence of intralesional haemorhage (Figure 3) and regional lyphadenopathy was more common in malignant lesions than benign lesions with statistically significant difference ( $P$ value 0.01 and $<0.05$ respectively). On the other hand the lesion size, the presence of cystic changes and the presence of pelvic fluid showed no significant difference between the benign and malignant groups. 
Table 3. Conventional MRI findings in the 78 patients.

\begin{tabular}{|c|c|c|c|c|c|c|c|}
\hline & \multicolumn{2}{|c|}{ Endometrial } & \multicolumn{2}{|l|}{ Myometrial } & \multicolumn{2}{|l|}{ Cervical } & \multirow{2}{*}{ P value } \\
\hline & benign & Malignant & Benign & Malignant & Benign & Malignant & \\
\hline Size (mm) & $14.2+4.6$ & $35.7 \mathrm{j} \pm 5.4$ & $104.3+4.3$ & $12.5+3.4$ & $21.3+3.4$ & $45.3+5.4$ & 0.54 \\
\hline \multicolumn{8}{|l|}{ Outline: } \\
\hline Regular & $20(90.9 \%)$ & $1(10 \%)$ & $19(82.6 \%)$ & $2(33.3 \%)$ & $11(84.6 \%)$ & & $<0.01$ \\
\hline Irregular & $2(9.1 \%)$ & $9(90 \%)$ & $4(17.4 \%)$ & $4(66.7 \%)$ & $2(15.4 \%)$ & $4(100 \%)$ & \\
\hline \multicolumn{8}{|c|}{ Cystic changes } \\
\hline Yes & $3(13.6 \%)$ & $3(30 \%)$ & $6(20.1 \%)$ & $3(50 \%)$ & $5(38.5 \%)$ & $2(50 \%)$ & 0.42 \\
\hline No & $19(86.4 \%$ & $7(70 \%)$ & $17(79.9 \%)$ & $3(50 \%)$ & $8(61.5 \%)$ & $2(50 \%)$ & \\
\hline \multicolumn{8}{|l|}{ T2 Signal } \\
\hline Low & $7(31.8 \%)$ & & $14(60.9 \%)$ & & $5(38.5 \%)$ & & \\
\hline Intermediate & $8(36.4 \%)$ & $2(30 \%)$ & $5(21.7 \%)$ & $1(16.7 \%)$ & & & $<0.05$ \\
\hline High & $6(22.3 \%)$ & $5(50 \%)$ & $2(8.7 \%)$ & $4(66.7 \%)$ & $5(38.5 \%)$ & $1(25 \%)$ & \\
\hline Hetrogenous & $2(9.1 \%)$ & $3(30 \%)$ & $2(8.7 \%)$ & $1(16.7)$ & $3(23.0 \%)$ & $4(75 \%)$ & \\
\hline \multicolumn{8}{|c|}{ Intralesional haemorrhage } \\
\hline Yes & $2(9.1 \%)$ & $8(80 \%)$ & $4(17.4 \%)$ & $2(33.3 \%)$ & $2(15.4 \%)$ & $3(75 \%)$ & 0.01 \\
\hline No & $20(90.9 \%)$ & $2(20 \%)$ & $19(82.6 \%)$ & $4(66.7 \%)$ & $11(84.6 \%)$ & $1(25 \%)$ & \\
\hline \multicolumn{8}{|l|}{ Pelvic fluid } \\
\hline Yes & $3(13.6 \%)$ & $2(30 \%)$ & $13(56.5 \%)$ & $5(33.3 \%)$ & $5(38.5 \%)$ & $2(50 \%)$ & 0.34 \\
\hline No & $19(86.4 \%)$ & $8(80 \%)$ & $10(43.5 \%)$ & $1(66.7 \%)$ & $8(61.5 \%)$ & $2(50 \%)$ & \\
\hline \multicolumn{8}{|c|}{ Regional lymph nodes } \\
\hline Yes & $2(9.1 \%)$ & $6(60 \%)$ & $4(17.4 \%)$ & $4(66.7 \%)$ & & $3(75 \%)$ & $<0.05$ \\
\hline No & $20(90.9 \%)$ & $440 \%)$ & $19(82.6 \%)$ & $2(33.3 \%)$ & $13(100 \%)$ & $1(25 \%)$ & \\
\hline
\end{tabular}
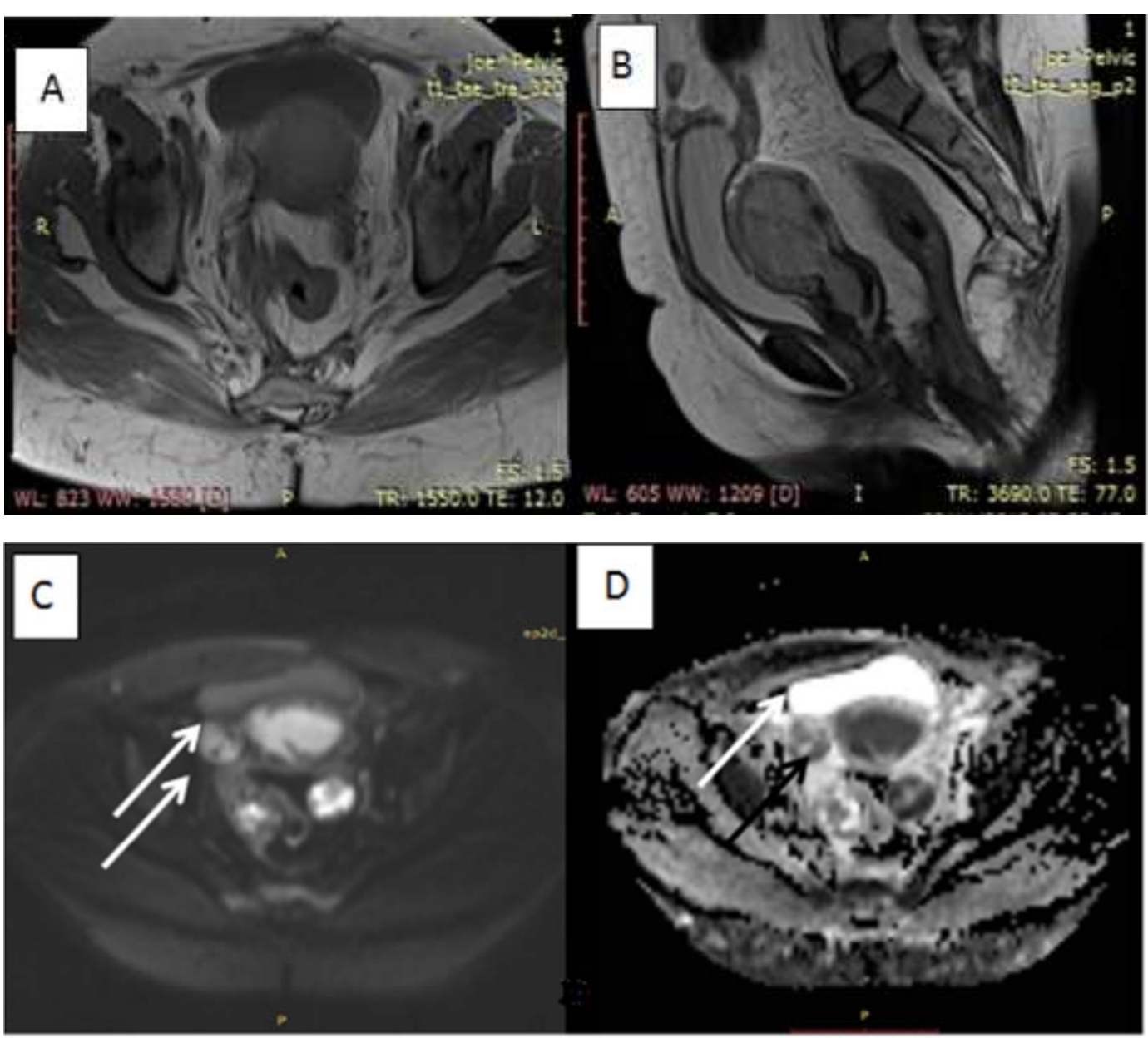

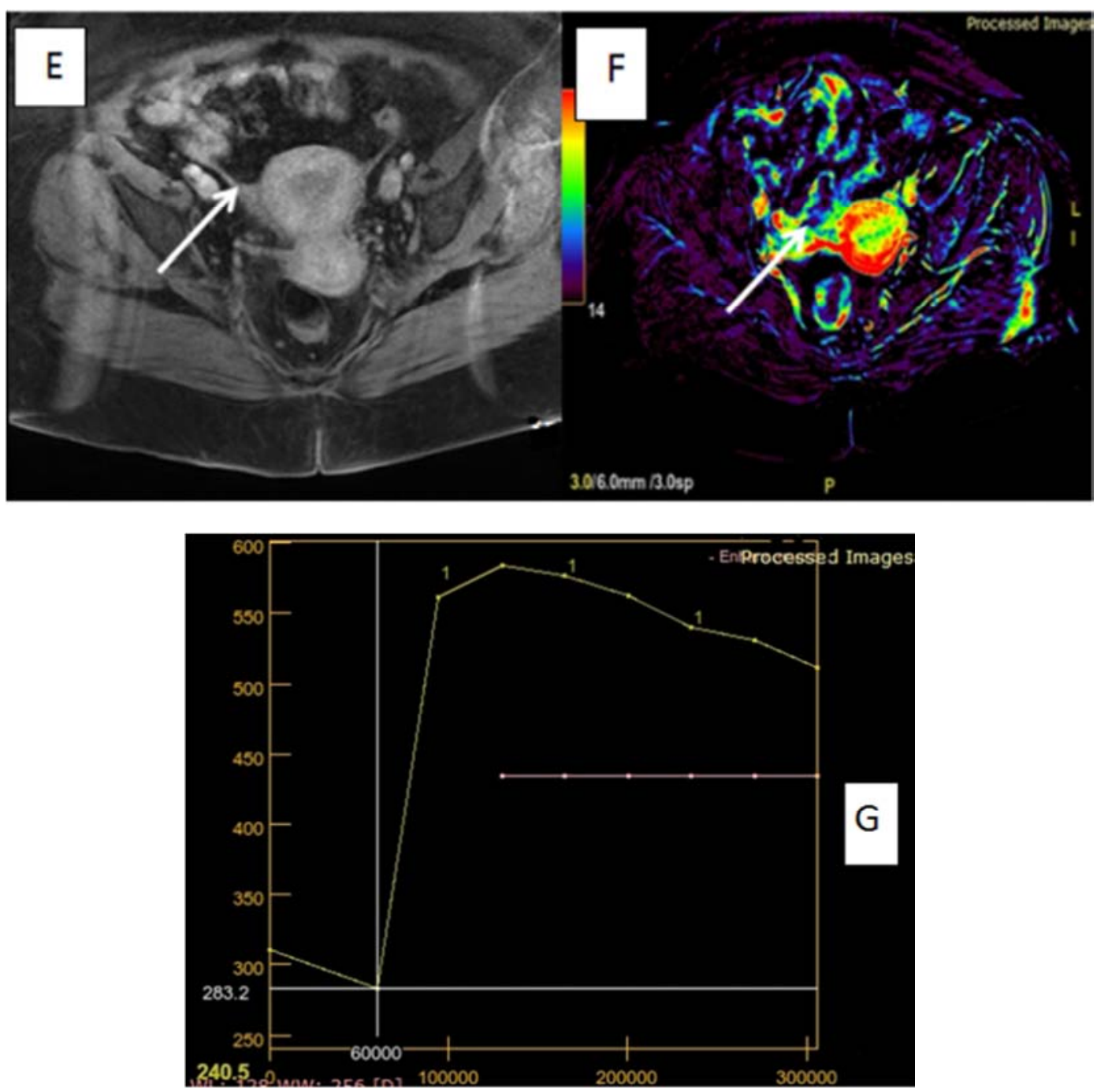

Figure 2. 65 years old female patient presented with excessive vaginal bleeding. (A\&B) Axial T1WI and sagittal T2WI showed endometrial soft tissue mass appearing isointense in T1 WI and high SI at T2SI. $(C \& D)$ Axial DWI ( $b$ value $=800)$ showed restricted DWI of endometrial mass lesion. ADC map showed low signal of the lesion with low ADC values $\left(0.95 \times 10^{-3} \mathrm{~mm}^{2} / \mathrm{sec}\right)$. (E, $\left.F \& G\right)$ Dynamic study through axial T1 fat sat post contrast showed rapid and early enhancement of the endometrial mass and rapid wash out, post processing color map and post processing time intensity curve showed type III curve (rapid enhancement of the mass with rapid wash out) with EA 273, MS 11, and THR $31 \mathrm{~mm} / \mathrm{sec}$. Final pathological diagnosis: undifferentiated endometrial adenocarcinoma.

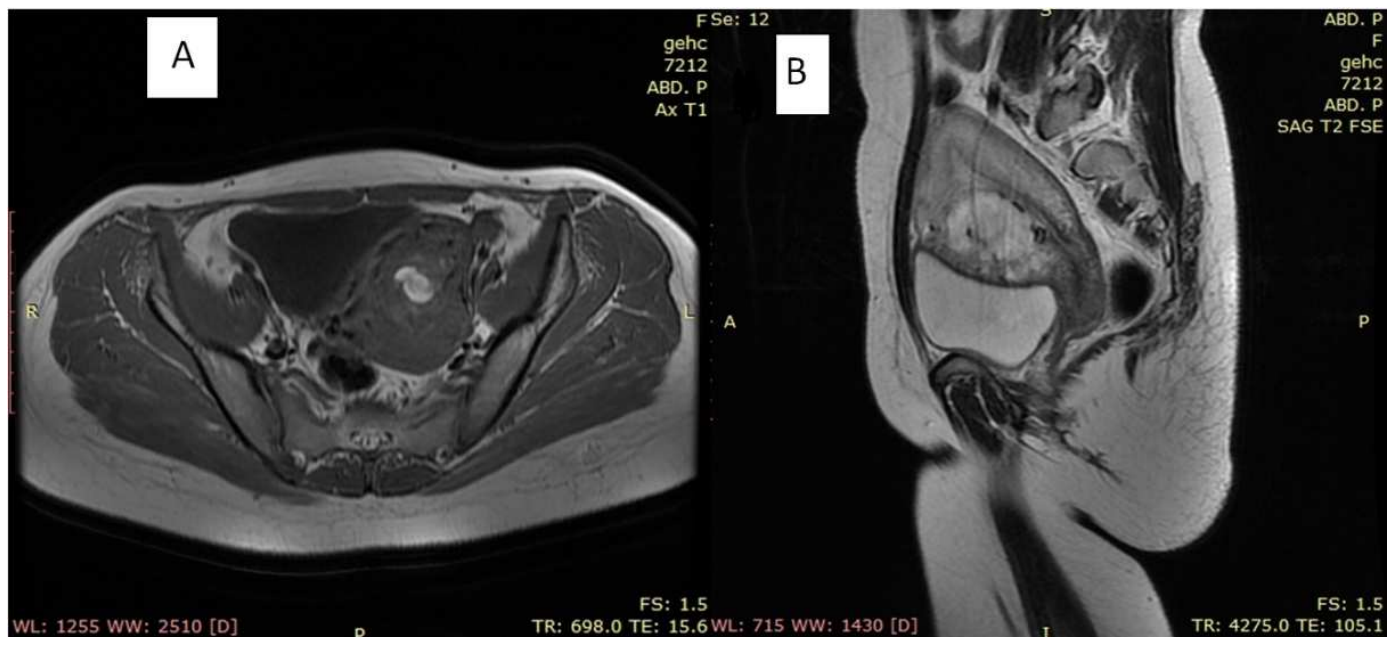



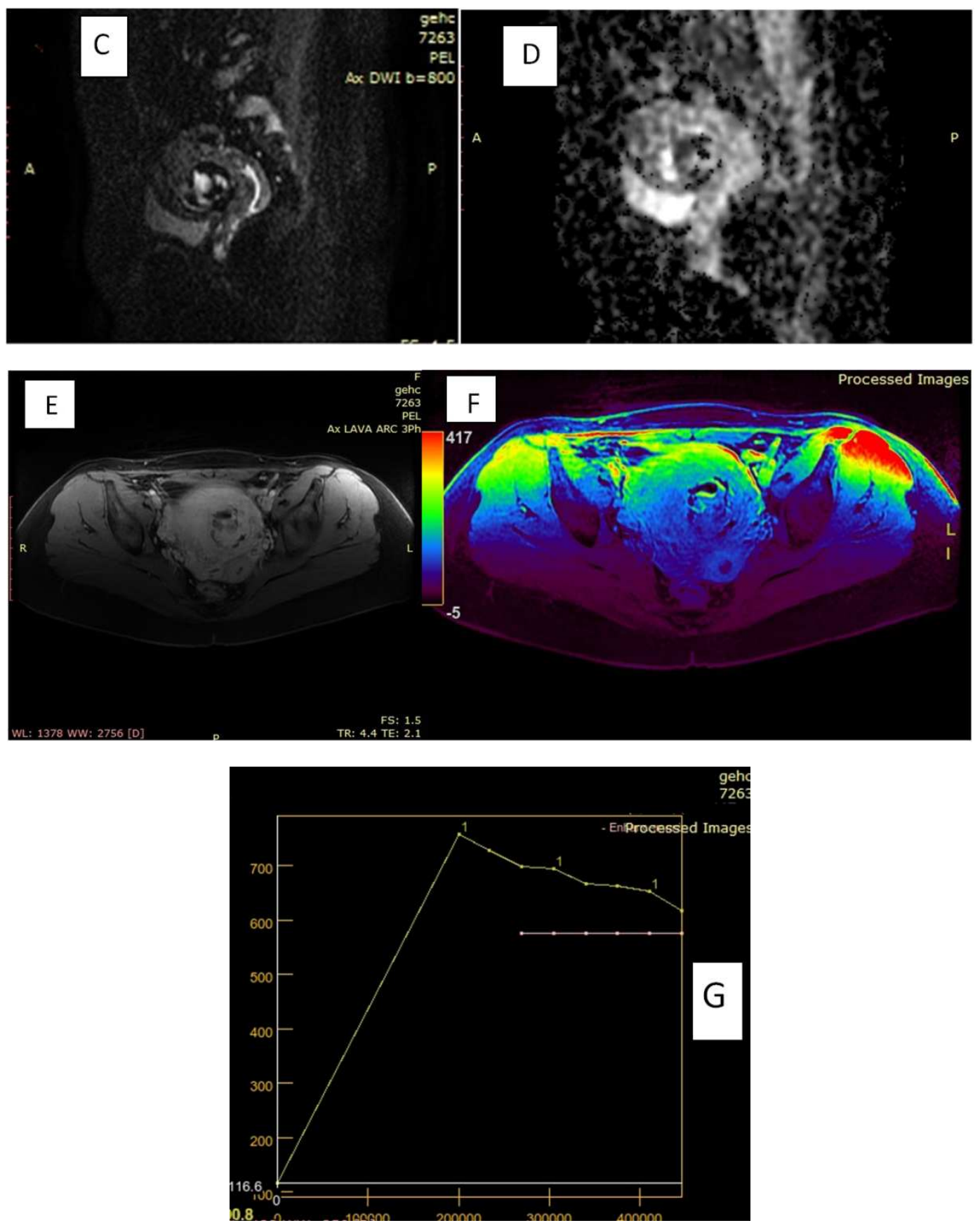

Figure 3. Female aged 34 years presented with excessive vaginal bleeding with high blood BHCG. A\&B) Axial T1WI \& sagittal Axial T2WI showed isointense signal intensity of anterior uterine wall mass with central high signal intensity denoting subacute hemorrhage, The mass shows high signal encroaching upon the endometrial cavity. $C \& D$ : Sagittal DWI ( $b$ value $=800$ ) showed heterogeneously restricted diffusion of the anterior uterine wall mass lesion. The ADC map showed low signal of the lesion with ADC values $\left(0.72 \times 10^{-3} \mathrm{~mm}^{2} / \mathrm{sec}\right)$. E. F\& G: Dynamic study through axial T1 fat sat post contrast showed rapid and early enhancement of the anterior uterine wall mass, Post processing color map and post processing time intensity curve showed type III curve (rapid enhancement of the mass with rapid wash out) with EA 300, MS 11, and THR $27 \mathrm{~mm} / \mathrm{sec..}$ Pathology: Anterior uterine wall choriocarcinoma.

\subsection{Diffusion Weighted Imaging (Tables 4, 5 \&6)}

Most malignant endometrial lesions showed restricted diffusion $(71.4 \%$ of endometrial carcinoma and $66.6 \%$ of choriocarcinoma), with only 1 false positive result (Figure 4). On the other hand, all benign lesions (except one) showed non restricted diffusion, with either hypointense signal or isointense signal, with statistically significant difference $(\mathrm{P}=<0.05)$.

In the current study, we had six pathologically proven sarcoma, of which 5 showed high signal on DWI (restricted diffusion). Three myomas (21.4\%) showed restricted diffusion. Most benign myomas showed hypointense signal (Figure 5) on DWI $(8 / 14,57.1 \%)$. The study included 4 cervical carcinoma, three of which showed restricted diffusion (75\%). All cervical myomas in the current study showed non restricted diffusion.

The mean ADC value of benign endometrial and myometrial lesions was higher significantly from malignant lesions ( $P$ value $<0.001$ and $<0.001$ ). The cut off value of $<1.1 \times 10^{-3} \mathrm{~mm}^{2} / \mathrm{sec}$ as a predictor of malignant lesions yielded asensitivity, specificity, 
PPV, NPV and accuracy of $89.65 \%, 91.54 \%, 88.67 \%, 92.41 \%$ and $95.21 \%$ respectivelt in the diagnosis of malignant endometrial lesions. For myometrial masses, the same cut off value yielded sensitivity, specificity, PPV, NPV and accuracy of $81.42 \%, 87.21 \%, 76.67 \%, 91.31 \%$ and $88.87 \%$ respectively in the prediction of malignant myometrial lesions (Figure 1). We used the same cut off value of $<1.1 \times 10^{-3} \mathrm{~mm}^{2} / \mathrm{sec}$ as a predictor of malignant cervical masses, with sensitivity, specificity, PPV, NPV and accuracy of $80.21 \%, 93.31 \%, 81.56 \%, 93.21 \%$ and $90.32 \%$ respectively.

Table 4. Signal intensity of different uterine lesions on DWI.

\begin{tabular}{llll}
\hline & \multicolumn{2}{l}{ Signal intensity on DWI } & \\
\cline { 2 - 4 } & Hyperintense & Isointense & Hypointense \\
\hline Endometrial lesions & & & \\
Endometrial hyperplasia & $0 / 13(0.00 \%)$ & $7 / 13(53.8 \%)$ & $5 / 13(38.4 \%)$ \\
Endometrial polyp & $1 / 9(11.1 \%)$ & $3 / 9(33.3 \%)$ & $5 / 9(55.5 \%)$ \\
Endometrial carcinoma & $5 / 7(71.4 \%)$ & $1 / 7(14.2 \%)$ & $1 / 7(14.2 \%)$ \\
Choriocarcinoma & $2 / 3(66.6 \%)$ & $1 / 3(33.3 \%)$ & $0 / 3(0.00 \%)$ \\
Myometrial & & & \\
Myoma & $3 / 14(21.4 \%)$ & $3 / 14(21.4 \%)$ & $8 / 14(57.1 \%)$ \\
Adenomyoma & $0 / 9(0.00 \%)$ & $1 / 9(11.1 \%)$ & $8 / 9(88.9 \%)$ \\
Sarcoma & $5 / 6(83.3 \%)$ & $1 / 6(16.7 \%)$ & $0 / 6(0.00 \%)$ \\
Cervical & & & \\
Myoma & $0 / 5(0 \%)$ & $1 / 5(20 \%)$ & $4 / 5(80 \%)$ \\
Polyp & $1 / 8(12.5 \%)$ & $2 / 8(25 \%)$ & $5 / 8(62.5 \%)$ \\
Carcinoma & $3 / 4(75 \%)$ & $1 / 4(25 \%)$ & $0 / 4(0 \%)$ \\
\hline
\end{tabular}

Table 5. ADC value in the different lesions.

\begin{tabular}{|c|c|c|c|c|}
\hline & \multicolumn{3}{|c|}{ ADC values of the uterine mass $\times 10^{-3} \mathrm{~mm}^{2} / \mathrm{sec}$. } & \multirow[t]{2}{*}{$\mathbf{P}$} \\
\hline & Min.-Max. & Mean \pm SD. & Median & \\
\hline \multicolumn{5}{|l|}{ Endometrial lesions } \\
\hline Endometrial hyperplasia & $0.93-1.52$ & $1.21 \pm 0.21$ & 1.24 & \\
\hline Endometrial polyp & $0.82-1.65$ & $1.31 \pm 0.32$ & 1.27 & $<0.001$ \\
\hline Endometrial carcinoma & $0.65-1.02$ & $0.82 \pm 0.39$ & 0.85 & \\
\hline Choriocarcinoma & $0.71-0.94$ & $0.79 \pm 0.46$ & 0.82 & \\
\hline \multicolumn{5}{|l|}{ Myometrial } \\
\hline Myoma & $0.83-2.82$ & $1.61 \pm 1.11$ & 1.74 & \\
\hline Adenomyoma & $1.02-1.95$ & $1.41 \pm 0.62$ & 1.49 & $<0.001$ \\
\hline Sarcoma & $0.85-1.22$ & $0.87 \pm 0.42$ & 0.88 & \\
\hline \multicolumn{5}{|l|}{ Cervical } \\
\hline Myoma & $0.98-1.82$ & $1.41 \pm 0.57$ & 1.45 & \\
\hline Polyp & $0.78-1.35$ & $1.29 \pm 0.81$ & 1.31 & $<0.001$ \\
\hline Carcinoma & $0.69-0.98$ & $0.78 \pm 0.34$ & 0.80 & \\
\hline
\end{tabular}

$\mathrm{p}$ : $\mathrm{p}$ value for comparing between the studied groups

*: Statistically significant at $\mathrm{p} \leq 0.05$

Table 6. Diagnostic accuracy of DWI \& ADC in differentiation between benign and malignant masses in the current study.

\begin{tabular}{lccccccc}
\hline & sensitivity & Specificity & PPV & NPP & c2 & FEp & Accuracy \\
\hline Endometrial masses & & & & & & & \\
DWI (Qualitative) & $85.71 \%$ & $96.55 \%$ & $85.71 \%$ & $96.55 \%$ & 0.0002 & 0.0001 & $94.44 \%$ \\
ADC (Quanitative) & $89.65 \%$ & $91.54 \%$ & $88.67 \%$ & $92.41 \%$ & 0.0002 & 0.0001 & $95.21 \%$ \\
Myometrial masses & & & & & & & \\
DWI (Qualitative) & $83.33 \%$ & $86.96 \%$ & $62.50 \%$ & $95.24 \%$ & 0.003 & 0.001 & $86.21 \%$ \\
ADC (Quanitative) & $81.42 \%$ & $87.21 \%$ & $76.67 \%$ & $91.31 \%$ & 0.002 & 0.001 & $88.87 \%$ \\
Cervical masses & & & & & & & \\
DWI (Qualitative) & $75.00 \%$ & $92.31 \%$ & $75.00 \%$ & $92.31 \%$ & 0.002 & 0.001 & $88.42 \%$ \\
ADC (Quanitative) & $80.21 \%$ & $93.31 \%$ & $81.56 \%$ & $93.21 \%$ & 0.002 & 0.001 & $90.32 \%$ \\
\hline
\end{tabular}

$\chi^{2}$ : Chi square testFE: Fischer exact $t$

$\mathrm{p}$ : $\mathrm{p}$ value for comparing between the studied groups

*: Statistically significant at $\mathrm{p} \leq 0.05$ 

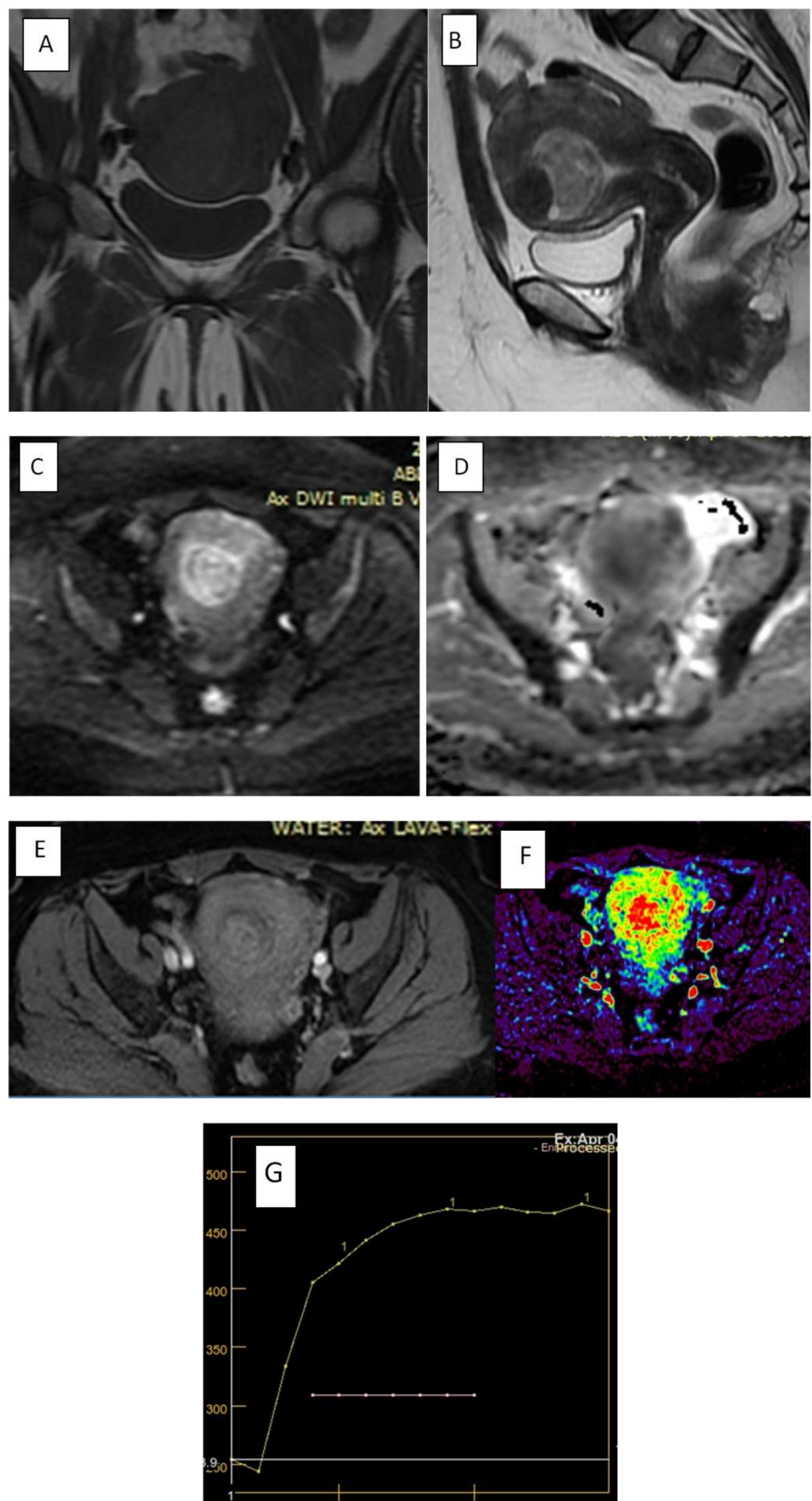

Figure 4. Female aged 42 years presented with vaginal bleeding. A\&B) coronalT1WI \& sagittal Axial T2WI showed a well defined large broad based endometrial polypwith hypointense signal on T1 WI and hyperintense signal on T2 W1. C\& D: Axial DWI and ADC map (b value =800) showed mildlyrestricted diffusion of the endometrial polyp. The ADC map showed low signal of the lesion with ADC values $\left(0.82 \times 10^{-3} \mathrm{~mm}^{2} / \mathrm{sec}\right) . \mathrm{E} . \mathrm{F}$ \& G: Dynamic study through axial T1 fat sat post contrast showed mild enhancement of the mass, Post processing color map and post processing time intensity curve showed type II curve (rapid enhancement of the mass with plateau like curve) with EA 290, MS 12, and THR $28 \mathrm{~mm} / \mathrm{sec}$. Pathology: benign endometrial polyp. 

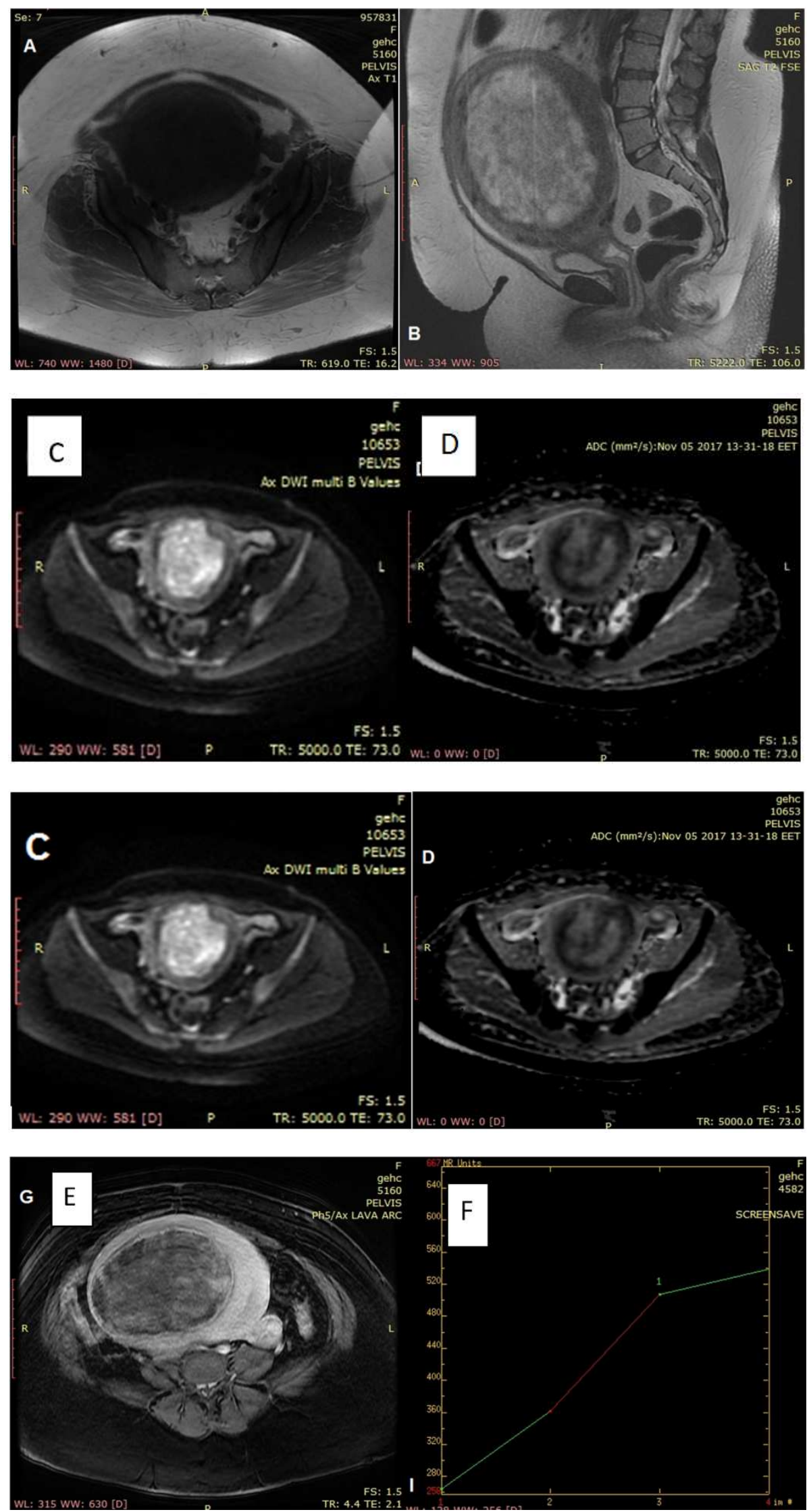

Figure 5. Patient aged 55 years with vaginal bleeding $(A \& B)$ Axial TIWI and sagittal T2WI showed large smoothly marginatedsoft tissue mass hypointense in TI WI and high SI at T2SI. C\&D Axial DWI (b value $=800)$ showed heterogeneous restricted DWI of endometrial mass lesion. ADC map showed heterogeneous signal of the lesion with $A D C$ value $\left(1.7 \times 10^{-3} \mathrm{E}, \mathrm{F}\right.$ : heterogeneous enhancement of the mass, and post processing time intensity curve showed type I curve (gradual and progressive enhancement of the mass) with EA 183, MS 8, and THR $55 \mathrm{~mm} / \mathrm{sec}$. The final pathological diagnosis was degenerated submucous fibroid. 


\subsection{Dynamic Contrast Enhanced MRI}

Qualitative DCE MRI:

Most benign endometrial lesions had Type I curve (17/22, $77.2 \%)$, with only one lesion showed type III curve (4.5\%). Ninety percent of malignant lesion had type III curve. The difference was statistically different $(\mathrm{P}<0.001)$.

Four benign myomas had type III curve, but 17 myomas showed type I curve. All malignant sarcomas had Type III curve (Table 7).

Of the 4 cervical carcinoma encountered in the study, 3 (75\%) had type III curve, an one had Type II curve. Most of the benign cervical lesions had type I curve $(9 / 13,69.2 \%)$, and only one lesion had type III curve (7.7\%). The difference between the benign and malignant lesions was statistically significant (0.001) (Figure 6).

Table 7. Types of curves in different lesions in dynamic contrast enhanced MRI.

\begin{tabular}{|c|c|c|c|c|c|}
\hline & \multicolumn{3}{|l|}{ Types of curves } & \multirow{2}{*}{${ }^{\mathrm{FE}} \mathbf{p}$} & \multirow{2}{*}{$\chi^{2}$} \\
\hline & Type I & Type II & Type III & & \\
\hline \multicolumn{6}{|l|}{ Endometrial lesions } \\
\hline Benign lesions & $17 / 22(77.2 \%)$ & $4 / 22(18.1 \%)$ & $1 / 22(4.5 \%)$ & 0.001 & 10.801 \\
\hline Malignant lesions & $0 / 10(0.00 \%)$ & $1 / 10(10.0 \%)$ & $9 / 10(90.0 \%)$ & & \\
\hline \multicolumn{6}{|l|}{ Myometrial } \\
\hline Benign lesions & $16 / 23(69.6 \%)$ & $3 / 23(13.04 \%)$ & $4 / 23(17.4 \%)$ & $0.003 *$ & $10.811^{*}$ \\
\hline Malignant lesions & $0 / 6(0.00 \%)$ & $0 / 6(16.7 \%)$ & $6 / 6(100 \%)$ & & \\
\hline \multicolumn{6}{|l|}{ Cervical } \\
\hline Benign lesions & $9 / 13(69.2 \%)$ & $3 / 13(23.1 \%)$ & $1 / 13(7.7 \%)$ & 0.001 & 10.755 \\
\hline Malignant lesions & $0 / 4(0 \%)$ & $1 / 4(25 \%)$ & $3 / 4(75 \%)$ & & \\
\hline
\end{tabular}
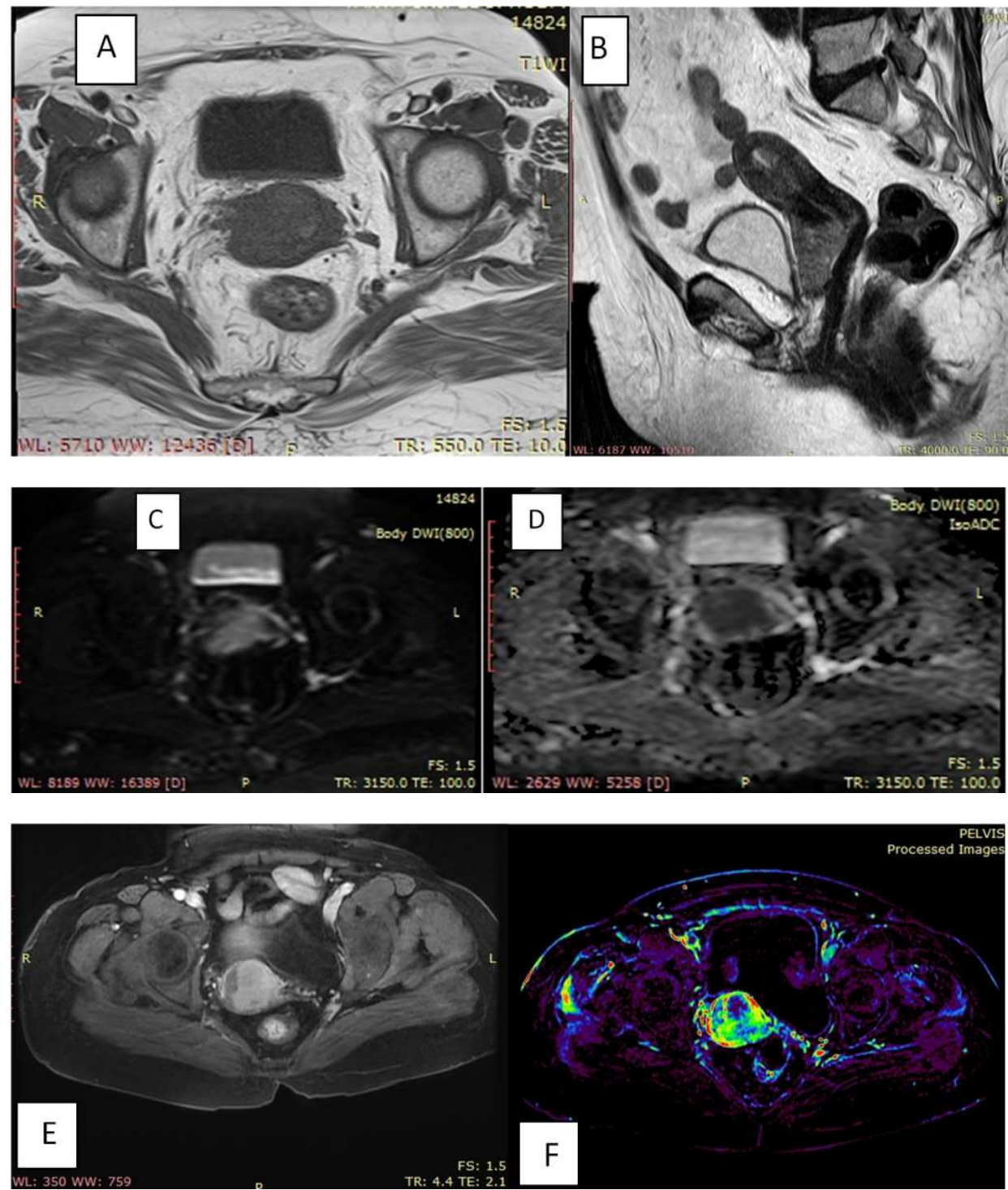


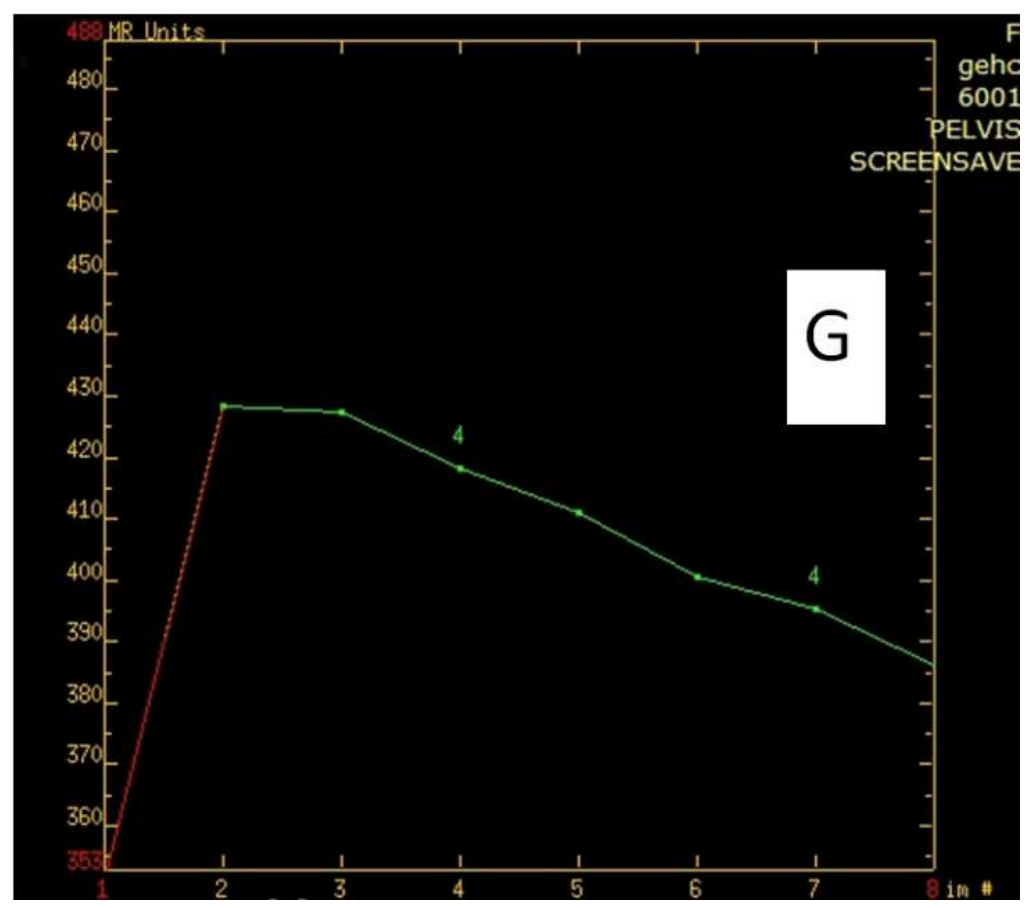

Figure 6. Patient aged 65 years with vaginal bleeding $(A \& B)$ Axial T1WI \& sagittal T2WI showed isointense signal endocervical mass in T1WI and high signal in sagittal T2WI. C\&D Axial DWI (b value $=800)$ showed restricted DWI of endocervical mass D): ADC map showed low signal of the lesion with ADC values $\left(0.90 \times 10^{-3} \mathrm{~mm}^{2} / \mathrm{sec}\right)$. E, $\left.F \& G\right)$ Dynamic contrast study through axial $T 1$ fat sat showed rapid and early enhancement of the cervical mass and rapid wash out, post processing color map and post processing time intensity curve showed type III curve (rapid enhancement of the mass with rapid wash out) with EA 270, MS 12, and THR $30 \mathrm{~mm} / \mathrm{sec}$. Final pathological diagnosis: Cervical carcinoma.

\section{Quantitative DCE MRI:}

In the current study the enhancement amplitude (EA), maximum slope (MS) and time of half rising (THR), all showed significant difference between the benign and malignant lesion in endometrial, myometrial and cervical lesions (Table 8). The enhancement amplitude for benign endometrial lesions was 226.20 which is significantly different from the malignant lesions $270.25(\mathrm{P}=<0.01)$. The maximum slope in benign endometrial lesions was 6.83 , and in malignant endometrial lesions $11.42(\mathrm{P}<0.001)$.

In general the accuracy of the DCE MRI in the differentiation between benign and malignant masses was $90.23 \%$ in endometrial masses, $88 \%$ in myometrial masses and $92.43 \%$ in cervical masses (table 9 ).

Table 8. Comparison of semiquantative values of dynamic contrast enhanced between the benign and malignant masses.

\begin{tabular}{llll}
\hline & Parameters (mean + SD) & & THR \\
\cline { 2 - 4 } & EA & MS & $57.43 \pm 11.02$ \\
\hline Endometrial lesions & $226.20 \pm 20.83$ & $6.83 \pm 3.44$ & $34.75 \pm 10.28$ \\
Benign lesions & $270.25 \pm 42.70$ & $11.42 \pm 0.24$ & $<0.011$ \\
Malignant lesions & $<0.01$ & $<0.001$ & $51.51 \pm 9.19$ \\
P value & & & $32.55 \pm 9.18$ \\
Myometrial & $238.26 \pm 31.72$ & $5.93 \pm 4.14$ & $<0.001$ \\
Benign lesions & $281.12 \pm 52.54$ & $12.32 \pm 0.84$ & $53.28 \pm 12.02$ \\
Malignant lesions & $<0.001$ & $<0.001$ & $32.34 \pm 11.21$ \\
P value & & & $<0.001$ \\
Cervical & $224.11 \pm 18.73$ & $7.23 \pm 2.56$ & $10.42 \pm 0.16$ \\
Benign lesions & $273.12 \pm 35.68$ & $<0.001$ & \\
Malignant lesions & $<0.001$ & & \\
Pa value &
\end{tabular}

EA: enhancement amplitude. MS maximum slope, THR: time of half rising

Table 9. Doagnostic accuracy of Dynamic contrast enhanced MRI in differentiation between benign and malignant lesions.

\begin{tabular}{llllllll}
\hline & sensitivity & Specificity & PPV & NPP & $\chi^{2}$ & ${ }^{\text {FE }}$ & Accuracy \\
\hline Endometrial masse & $91.60 \%$ & 88.67 & 94.86 & 94.86 & $10.811^{*}$ & $0.003^{*}$ & 90.23 \\
Myometrial masses & $100 \%$ & 82.72 & 92.23 & 92.23 & $10.711^{*}$ & $0.001^{*}$ & 87 \\
Cervical masses & $92.20 \%$ & 89.91 & 95.81 & 95.81 & $10.811^{*}$ & $0.003^{*}$ & 92.43 \\
\hline
\end{tabular}




\section{Discussion}

Our results emphasized the important role of DWI and DCE-MRI in the pre-operative diagnosis of endometrial, myometrial and cervical lesions.

Endometrial lesions represent a diagnostic challenge because of the overlap in the sonographic and MRI appearance between benign and malignant lesions [27, 28]. In the current study, most malignant lesions had irregular outline $(90 \%)$, high or intermediate signal on T2 WI $(80 \%)$, while benign endometrial lesions had regular outline (90.9\%), and either low or intermediate signal (68.2\%). Our results are close to those of Kierans et al [27] who reported the irregular myometrial-endometrial interface as the most differentiating sign between benign and malignant endometrial lesions. Most endometrial malignancies (70\%) showed hyperintense signal on DWI, with mean ADC value, with the mean $\mathrm{ADC}$ value for endometrial carcinoma was $0.82 \pm 0.39 \times 10^{-3} \mathrm{~mm}^{2} / \mathrm{s}$ which is lower than the mean value of endometrial hyperplasia and endometrial polyp $\left(1.21+0.21 \times 10^{-3} \mathrm{~mm}^{2} / \mathrm{s}\right.$, and $1.31+0.32 \times 10^{-3} \mathrm{~mm}^{2} / \mathrm{s}$ respectively). The accuracy of quantitative and qualitative DWI in the current was higher other modalities and higher than the DWI accuracy in myometrial or cervical lesions. Our results are in agreement with those of Fuji et al (28), who reported sensitivity, specificity and accuracy of $84.6 \%, 100 \%$ and $92 \%$ respectively in a study included 25 patients. Also, Elsammak et al (29), in a study included 42 patients reported a significantly low ADC value in malignant lesions than benign lesions $\left(0.82+1.09 \times 10^{-3} \mathrm{~mm}^{2} / \mathrm{s} \quad\right.$ Vs $1.44+0.15 \times 10^{-3} \mathrm{~mm}^{2} / \mathrm{s}$ respectively).

Also, our results showed that the DWI is more accurate than DCE-MRI in the diagnosis of endometrial carcinoma (95.21\% Versus 90.23\%). Beddy et al [30] compared the performance of DWI and DCE-MRI in staging of endometrial carcinoma. They reported that DWI is more accurate in staging and detection of myometrial invasion than DCE-MRI. They explained their results by the presence of associated leimomyoam or adenomosis, loss of tumor to myometrium contrast, poor definition of junctional zone, and extension of the tumor to the cornua, and they stated this factors may affect DCE-MRI than DWI.

In the current study, 5 sarcomas (83.3\%) showed hyperintense signal on DWI, with the mean ADC value $0.87 \pm 0.42 \times 10^{-3} \mathrm{~mm}^{2} / \mathrm{sec}$, which significantly lower than that of myomas $1.61 \pm 1.11 \times 10^{-3} \mathrm{~mm}^{2} / \mathrm{sec}$. However, $21.3 \%$ of myomas shows hyperintense signal on DWI. The accuracy of DWI in differentiation between myomas and sarcomas was $86.21 \%$. The restricted diffusion in malignant tissues is caused by hypercellularity, irregular nuclear outline and large nuclei which result in limitation of water molecules movements [31-33]. Tamai et al [34] reported that some cellular myomas may not be differentiated from sarcomas based on DWI and ADC values. In the current study most of myomas (58.1\%) showed hypointense signal. The low signal on DWI of the myomas is explained by the T2 blackout effect which indicates hypointense signal on DWI due to hypointense signal on T2 WI $[35,36]$. The ADC value of myomas may show wide range reflecting the variability in cellularity and presence of hyalainized tissue within the myomas [37]. Also, the presence of non parallel collagen fibers may be a cause of the restricted diffusion observed in some myomas [38].

In the current study, we used semi-quantitative parameters which are less accurate than quantitative parameters but they are easily evaluated, more applicable in daily practice, and do not need complicated post processing [39].

In the current study all sarcomas had type III time intensity curve (malignant type with rapid washout), on the other hand most myomas (69.6\%) had type I curve, with statistically significant difference $(\mathrm{P}=0.003)$. Our results are not similar to those of Thomassin-Naggara et al [40], who reported type III curve in $4 / 18(22.2 \%)$ in malignant group, the cause of difference between our results and their results is that the malignant group in their study contained malignant and border line lesions. Shimada et al [22] in a study included 30 patients with uterine myomas found the degree of hyalinization inversely proportionate to the degree of enhanacement and intra-tumoral vessel density. Our results are approximate to those of Tamburrini et al [41], who reported type III curve in all 8 malignant uterine sarcomas included in their study and reported four cases (of thirty four myomas) with benign myomas.

The uterine cervix malignancy is more common than uterine sarcoma, and is still a significant health problem in developing countries [42] The mean ADC value in malignant cervical masses $\left(0.78 \pm 0.34 \times 10^{-3} \mathrm{~mm}^{2} / \mathrm{sec}\right)$ was significantly lower than cervical myoma $\left(1.41 \pm 0.57 \times 10^{-3} \mathrm{~mm}^{2} / \mathrm{sec}\right)$, which is similar to the results of Kilickesmez et al [43] who reporeda mean ADC value of benign and malignant masses to be $\left(1.55 \pm 0.33 \times 10^{-3} \mathrm{~mm}^{2} / \mathrm{sec}\right.$ and $0.88 \pm 0.11 \times 10^{-3} \mathrm{~mm}^{2} / \mathrm{sec}$ respectively). Also they used a cut off value $1.05 \times 10^{-3} \mathrm{~mm}^{2} / \mathrm{sec}$, close to the cut off value $1.1 \times 10^{-}$ ${ }^{3} \mathrm{~mm}^{2} / \mathrm{sec}$ used in the current study and they reported asensitivity, specificity, and accuracy of $95.83 \%, 94.55 \%$, and $94.94 \%$.

In general, both DCE-MRI and DWI sequences provide functional informations abut changes in angiogenesis, perfusion, oxygenation, and tumour microstructure and can yield quantitative or semi-quantitative parameters which are a potential biomarker of tumour $[44,45]$.

There are some limitations in our study. First, the small sample size of the uterine sarcoma. Second, the study population was partially selected and consisted of non-consecutive patients. Third, menstrual phase and the menstrual state (whether pre-menpausal or postmenopausal) of the patients was not recorded and correlated with the results.

\section{Conclusion}

In clinically and sonographically indeterminate endometrial, myometrial or cervical masses, DWI and DCEMRI proved to be more accurate than conventional MRI in differentiation between benign and malignant masses, with 
the DWI and ADC value had more sensitivity and specificity especially in endometrial masses and should be included in the routine pelvic MRI. DCE-MRI should be reserved for still questionable cases after DWI and to study the vascularity of the lesions.

\section{Appendix}

Table A1. Different lesions encountered in the study.

\begin{tabular}{lll}
\hline & No & Age mean \pm SD \\
\hline Endometrial lesions & & \\
Endometrial hyperplasia & 13 & $38.4 \pm 6.7$ years \\
Endometrial polyp & 9 & $42.3 \pm 5.4$ years \\
Endometrial carcinoma & 7 & $51.7 \pm 5.4$ years \\
Choriocarcinoma & 3 & $25.5 \pm 2.7$ years \\
Myometrial & & \\
Myoma & 14 & $33.5 \pm 7.8$ years \\
Adenomyoma & 9 & $39.6 \pm 5.4$ years \\
Sarcoma & 6 & $50.3 \pm 6.2$ years \\
Cervical & & \\
Myoma & 5 & $31.3 \pm 5.4$ years \\
Polyp & 8 & $29.7 \pm 3.3$ years \\
Carcinoma & 4 & $56.3 \pm 3.2$ years \\
\hline
\end{tabular}

Table A2. Parameters of MRI sequences in the current study.

\begin{tabular}{llllll}
\hline & Axial T2 & Sagittal T2 & Cornonal T2 & Axial T1 & DWI \\
\hline Matrix & $512 \times 187$ & $51 \times 245$ & $512 \times 245$ & $245 \times 145$ & 12 \\
Flip angle & - & - & - & 7 & 90 \\
Slice thickness & 7 & 5 & 5 & 5 & 1.5 \\
Slice Spacing & 1.5 & 1 & $256 \times 256$ & 40 \\
Slice number & 35 & 25 & 25 & $450-600$ \\
TR (ms) & $5000-6000$ & $4500-7000$ & $4500-7000$ & 1 \\
TE (ms) & $100-120$ & $110^{-1} 20$ & $110^{-1} 20$ & $5500-6500$ \\
\hline
\end{tabular}

[7] Amant F, Coosemans A, Debiec-Rychter M, et al. Clinical management of uterine sarcomas. Lancet Oncol. 2009; 10: 1188. http://dx.doi.org/10.1016/S1470-2045 (09) 70226-8.

\section{References}

[1] Jemal A, Bray F, Center MM, Ferlay J, Ward E, and Forman D, "Global cancer statistics," CA: A Cancer Journal for Clinicians, vol. 61, no. 2, pp. 69-90, 2011.

[2] Forman D, Bray F, Brewster D et al., Cancer Incidence in Five Continents, Vol. X. Cancer Incidence in Five Continents, IARC, Lyon, France, 2013.

[3] Freedman LS, Edwards B, Ries L, and Young J, Cancer Incidence in Four Member Countries (Cyprus, Egypt, Israel, and Jordan) of the Middle East Cancer Consortium (MECC) Compared with US SEER. Cancer Incidence in Four Member Countries (Cyprus, Egypt, Israel, and Jordan) of the Middle East Cancer Consortium (MECC) Compared with US SEER, 2006.

[4] Alshahrani S, Soliman AS, Hablas A, et al. Changes in Uterine Cancer Incidence Rates in Egypt. Obstetrics and Gynecology International. Volume 2018, Article ID 3632067, 10 pages.

[5] Bonneau C. Value of ultrasonography and magnetic resonance imaging for the characterization of uterine mesenchymal tumors. Acta Obstetricia et GynecologicaScandinavica. 2014; 93 (3): 261-268.

[6] Dueholm M, Lundorf E, Hansen ES, et al. Accuracy of magnetic resonance imaging and transvaginal ultrasonography in the diagnosis, mapping, and measurement ofuterine myomas. Am J Obstet Gynecol. 2002; 186 (3): 409-15. PMid: 11904599 http://dx.doi.org/10.1067/mob.2002.121725

[8] Sahdev A, Sohaib SA, Jacobs I et al (2001) MR imaging of uterine sarcomas. AJR Am J Roentgenol 177: 1307-1311.

[9] Ueda H, Togashi K, Konishi I et al (1999) Unusual appearances ofuterine leiomyomas: MR imaging findings and their histopathologic backgrounds. Radiographics 19 Spec No: S131-S145.

[10] Qayyum A. Diffusion-weighted imaging in the abdomen and pelvis: concepts and applications. Radiogr Rev Publ Radiol Soc N Am Inc. 2009; 29: 1797-810.

[11] Nakamura K, Kajitani S, Joja I, Haruma T, Fukushima C, Kusumoto $\mathrm{T}$, et al. The post treatment mean apparent diffusion coefficient of primary tumour is superior to pretreatment ADC mean of primary tumour as a predictor of prognosis with cervical cancer. Cancer Med. 2013; 2: 519-25.

[12] Lyng H, Haraldseth O, Rofstad EK (2000) Measurement of cell density and necrotic fraction in human melanoma xenografts by diffusion weighted magnetic resonance imaging. Magn Reson Med 43: 828-836.

[13] Sumi M, Ichikawa Y, Nakamura T (2007) Diagnostic ability of apparent diffusion coefficients for lymphomas and carcinomas in the pharynx. Eur Radiol 17: 2631-263.

[14] Matoba M, Tonami H, Kondou T, Yokota H, Higashi K, Toga H, Sakuma T (2007) Lung carcinoma: diffusionweighted $\mathrm{mr}$ imaging-preliminary evaluation with apparent diffusion coefficient. Radiology 243: 570-577. 
[15] Matsuki M, Inada $Y$, Nakai G, Tatsugami F, Tanikake M, Narabayashi I, Masuda D, Arisaka Y, Takaori K, Tanigawa N (2007) Diffusion-weighed MR imaging of pancreatic carcinoma. Abdom Imaging 32 (4): 481-48.

[16] Jacobs MA, Herskovits EH, Kim HS (2005) Uterine fibroids: diffusion weighted MR imaging for monitoring therapy with focused ultrasound surgery-preliminary study. Radiology 236: 196-203.

[17] Matsuki M, Inada Y, Tatsugami F, Tanikake M, Narabayashi I, Katsuoka Y (2007) Diffusion-weighted MR imaging for urinary bladder carcinoma: initial results. Eur Radiol 17: 201204.

[18] Tasu K, Kuroki Y, Nawano S, Kuroki S, Tsukamoto T, Yamamoto S, Motoori K, Ueda T (2006) Hepatic metastases: diffusion-weighted sensitivity-encoding versus SPIOenhanced MR imaging. Radiology 239: 122-130.

[19] Naganawa S, Sato C, Kumada H, Ishigaki T, Miura S, Takizawa O (2005) Apparent diffusion coefficient in cervical cancer of the uterus: comparison with the normal uterine cervix. Eur Radiol 15: 71-78.

[20] Tamai K, Koyama T, Saga T, Umeoka S, Mikami Y, Fujii S, Togashi K (2007) Diffusion-weighted MR imaging of uterine endometrial cancer. J Magn Reson Imaging 26: 682-687.

[21] Liapi E, Kamel IR, Bluemke DA, Jacobs MA, Kim HS (2005) ssessment of response of uterine fibroids and myometrium to embolization using diffusion-weighted echoplanar MR imaging. J Comput Assist Tomogr 29: 83-86.

[22] Shimada K, Ohashi I, Kasahara I, Watanabe H, Ohta S, Miyasaka N, Itoh E, Shibuya H (2004) Differentiation between completely hyalinized uterine leiomyomas and ordinary leiomyomas: three-phase dynamic magnetic resonance imaging (MRI) vs diffusion weighted MRI with very small b-factors. J Magn Reson Imaging 20: 97-104.

[23] Gaustad JV, Brurberg KG, Simonsen TG, Mollatt CS, Rofstad EK. Tumor vascularity assessed by magnetic resonance imaging and intravital microscopy imaging. Neoplasia. 2008; 10 (4): 354-62. doi: 10.1593/neo. 08162.

[24] Kim YE, Lim JS, Choi J, Kim D, Myoung S, Kim MJ, et al. Perfusion parameters of dynamic contrast-enhanced magnetic resonance imaging in patients with rectal cancer: correlation with microvascular density and vascular endothelial growth factor expression. Korean J Radiol. 2013; 14 (6): 878-85. doi: 10.3348/kjr.2013.14.6.878.[PubMed: 24265562].

[25] Tofts PS, Brix G, Buckley DL, Evelhoch JL, Henderson E, Knopp MV. Estimating kinetic parameters from dynamic contrast-enhanced T 1-weighted MRI of a diffusable tracer: standardized quantities and symbols. J Magn Reson Imaging. 1999; 10 (3): 223-32.

[26] Choyke PL, Dwyer AJ, Knopp MV. Functional tumor imaging with dynamic contrast-enhanced magnetic resonance imaging. J Magn Reson Imaging. 2003; 17: 509-520. [PubMed: 12720260] 3. NCI. CIP guidelines.

[27] Kierans AS, G. L. Bennett, M. HaghighiUtility of conventional and diffusion-weighted MRI features in distinguishing benign from malignant endometrial lesions Eur J Radiol, 83 (4) (2013), pp. 726-732.

[28] Fujii S, E. Matsusue, J. Kigawa, et al. Diagnostic accuracy of the apparent diffusion coefficient in differentiating benign from malignant uterine endometrial cavity lesions initial results Eur Radiol, 18 (2) (2008), pp. 384-389.

[29] Elsammak A, Shehata SM, Abulezz M, Gouhar G: Efficiency of diffusion weighted magnetic resonance in differentiation between benign and malignant endometrial lesions. The Egyptian Journal of Radiology and Nuclear Medicine 48 (2017) 751-759.

[30] Beddy P, Moyle P, Kataoka M, Yamamoto AK, Joubert I, Lomas D, Crawford R, Sala E. evaluation of depth of myometrial invasion and overall staging in endometrial cancer: comparison of diffusion-weighted and dynamic contrastenhanced MR imaging. Radiology. 2012 Feb; 262 (2): 530-7. doi: 10.1148/radiol.11110984. Epub 2011 Nov 23.

[31] Szafer A, Zhong J, Gore JC (1995) Theoretical model for water diffusion in tissues. Magn Reson Med 33: 697-712.

[32] Liu KF, Li F, Tatlisumak T, Garcia JH, Sotak CH, Fisher M, Fenstermacher JD (2001) Regional variations in the apparent diffusion coefficient and the intracellular distribution of water in rat brain during acute focal ischemia. Stroke 32: 1897-1905.

[33] Knight RA, Ordidge R, Helpern J, Chopp M, Rodolosi L, Peck D (1991) Temporal evolution of ischemic damage in rat brain measured by proton nuclear magnetic resonance imaging. Stroke 22: 802-808.

[34] Tamai K, Koyama T, Saga T, Morisawa N, Fujimoto K, Mikami Y, Togashi K (2008) The utility of diffusion-weighted MR imaging for differentiating uterine sarcomas from benign leiomyomas. Eur Radiol 18: 723-730.

[35] Maldjian JA, Listerud J, Moonis G, Siddiqi F (2001) Computing diffusion rates in T2-dark hematomas and areas of low T2 signal. AJNR Am J Neuroradiol 22: 112-118.

[36] Hiwatashi A, Kinoshita T, Moritani T, Wang HZ, Shrier DA, Numaguchi Y, Ekholm SE, Westesson PL (2003) Hypointensity on diffusion-weighted MRI of the brain related to T2 shortening and susceptibility effects. AJR Am J Roentgenol 181: 1705-1709.

[37] Shimada K, Ohashi I, Kasahara I, Watanabe H, Ohta S, Miyasaka N, Itoh E, Shibuya H (2004) Differentiation between completely hyalinized uterine leiomyomas and ordinary leiomyomas: three-phase dynamic magnetic resonance imaging (MRI) vs. diffusionweighted MRI with very small b-factors. J Magn Reson Imaging 20: 97-104.

[38] Leppert PC, Baginski T, Prupas C, Catherino WH, Pletcher S, Segars JH. Comparative ultrastructure of collagen fibrils in uterine leiomyomas and normal myometrium. Fertil Steril 2004; 82 Suppl 3: 1182-1187 [PMID: 15474093].

[39] Kim YS, Kim BG, Rhim H, Bae DS, Lee JW, Kim TJ, Choi $\mathrm{CH}$, Lee YY, Lim HK.. Uterine fibroids: semiquantitative perfusion MR imaging parameters associated with the intraprocedural and immediate postprocedural treatment efficiencies of MR imaging-guided high-intensity focused ultrasound ablation. Radiology. 2014 Nov; 273 (2): 462-71. doi: 0.1148/radiol.14132719. Epub 2014 Jul 1.

[40] Thomassin-Naggara I, Dechoux S, Bonneau C, Morel A, Rouzier R, Carette MF, Daraï E, Bazot M. How to differentiate benign from malignant myometrial tumours using MR imaging. Eur adiol. 2013 Aug; 23 (8): 2306-14. doi: 10.1007/s00330-013-2819-9. Epub 2013 Apr 8. 
[41] Oscar Tamburrini, Claudia Smiraglio, Nicoletta Caputo, Nadia Mastroianni, Giuseppe Guarascio, Di Cello Annalisa, Michele Morelli, Fulvio ZulloI. The contribution of Magnetic Resonance Imaging in the differential diagnosis between leiomyoma typical, atypical and uterine sarcomas: Personal experience. International Journal of Diagnostic Imaging, 2016, Vol. 3, No. 2.

[42] Solomon D, Breen N, McNeel T. Cervical cancer screening rates in the United States and the potential impact of implementation of screening guidelines. CA Cancer J Clin 2007; 57: 105-111.
[43] Kilickesmez O, Bayramoglu S, Inci E, Cimilli T, Kayhan A. Quantitative diffusion-weighted magnetic resonance imaging of normal and diseased uterine zones. Acta Radiol 2009; 50: 340-347 [PMID: 19235579

DOI: $10.1080 / 02841850902735858]$.

[44] Harry VN, Semple SI, Parkin DE, Gilbert FJ. Use of new imaging techniques to predict tumour response to therapy. Lancet Oncol. 2010; 11 (1): 92-102.

[45] Punwani S. Diffusion weighted imaging of female pelvic cancers: concepts and clinical applications. Eur J Radiol. 2011; 78 (1): 21-9. 